\title{
MANIFESTATION OF THE OPTICAL STARK EFFECT IN DIFFERENTIAL TRANSMISSION SPECTRA
}

\author{
A. Savickas and E. Gaižauskas \\ Department of Quantum Electronics, Vilnius University, Saulètekio 9, LT-10222 Vilnius, Lithuania \\ E-mail: aurimas.savickas@ff.vu.lt
}

Received 14 February 2008; revised 3 April 2008; accepted 9 June 2008

\begin{abstract}
The two-level quantum system is applied in theoretical modelling of the angle-resolved differential absorption spectra induced by the strong near-resonant laser field in quantum dots. The simulations of induced polarization waves at appropriate directions have been performed by means of the density matrix formalism, without using perturbation theory. An interpretation of the differential spectra is provided referring to representation of the dressed states, with their energies experiencing Stark shifts due to the action of the laser field. The feasibility of the light and matter interaction strength characterization via observed Mollow spectrum at the different (from the probe beam) direction is demonstrated.
\end{abstract}

Keywords: femtosecond spectroscopy, differential spectra, quantum dots, Stark effect, four-wave mixing, Mollow spectrum, dressed states

PACS: 78.20.Jq, 78.47.+p, 78.67.Hc

\section{Introduction}

Progress in constructing optical switches, logical elements, as well as entries for quantum computing [1-3] increases demand for characterization of strong-field affected electronic states in quantum wells, quantum wires, and quantum dots (QD). In particular, there has been considerable interest in the investigation of the optical Stark effect (OSE), i. e. light-induced shift of energy levels in the presence of non-resonant laser fields in nanoscale materials [4]. Optical spectroscopy methods count among the most versatile and routine ones for characterizing strong-field limit of light-matter interaction in such kind of quantum systems. Among a number of experimental and theoretical studies of such kind, analysis of the strong-coupling regime between confined excitons and micro-cavity photons [5], dynamics of basic laser-induced coherences (that can be observed, e. g., in quantum beat spectroscopy), as well as Rabi oscillations in pump-probe spectroscopy $[6,7]$ should be noted.

The last technique is known as standard one for investigating both transient and stationary response of medium to the pump-induced perturbation. This method implicitly assumes weak probe beam, which measures induced absorption (transmission) changes. However, it should be stressed that, to reduce the com- plexity of the problem, the description of the technique discussed here is based on two different kinds of approximations, even for the relatively strong pump field. In the time-resolved case, negligible excited state population is approached, allowing perturbative treatment of system evolution $[9,8]$. On the other hand, countinuous wave (cw) approximation and appropriate to this case representation of the dressed states is used to consider OSE in the strong-field (high Rabi frequency) limit [10].

Because our interest in this work is the OSE manifestation in differential absorption spectra observable in the limit of strong pulsed excitation, none of the mentioned above approximations is suitable for our purposes. Therefore numerical integration of the Bloch equations is performed when obtaining the main results in this work, which is performed in the manner of angleand time-resolved pump-probe spectroscopy developed for description of the coherent effects in femtosecond pump-probe spectroscopy [11-16].

In this paper we stress the peculiarities of the differential pump-probe spectra resulting from the strongfield induced OSE in QD, owing to the fact that both long phase relaxation time and huge strength of optical transitions are characteristic of confined excitons. Because of the discrete atom-like lines of the excitonic 
spectra, here we restrict our consideration to two-level quantum system.

The paper is organized as follows. Section 2 provides our approach and main equation appropriate for the analysis of the angle-resolved pump-probe measurements. Section 3 presents the numerical modelling results and interpretations based on the dressed state picture. We conclude with discussion of expected benefits of using angle-resolved pump-probe configuration analysis of the OSE implemented peculiarities in differential absorption spectra in Sec. 4.

\section{Theoretical model and calculation procedure}

\subsection{Pump-probe spectroscopy in two-level approximation}

Consider a two-level system with a transition frequency $\omega_{21}$ subjected to the strong pump, which has carrying frequency $\omega_{\mathrm{L}}$ and wave vector $\mathbf{k}_{\mathrm{L}}$, and a weaker probe, characterized by frequency $\omega_{\mathrm{P}}$ and wave vector $\mathbf{k}_{\mathrm{P}}$, accordingly. Total electric field can be written as superposition of two quasi-monochromatic plane-wave pulses:

$$
\begin{aligned}
E(t)= & \frac{1}{2}\left[\varepsilon_{\mathbf{L}}(t) \mathrm{e}^{\mathrm{i}\left[\mathbf{k}_{\mathbf{L}} \mathbf{r}-\omega_{\mathrm{L}}\left(t-\tau_{\mathrm{L}}\right)\right]}\right. \\
& \left.+\varepsilon_{\mathrm{P}}(t) \mathrm{e}^{\mathrm{i}\left[\mathbf{k}_{\mathbf{P}} \mathbf{r}-\omega_{\mathrm{P}}\left(t-\tau_{\mathrm{P}}\right)\right]}+\text { c. c. }\right],
\end{aligned}
$$

where $\varepsilon_{\mathrm{L}}(t)$ and $\varepsilon_{\mathrm{P}}(t)$ are slowly varying envelopes of electric field, c. c. means complex conjugate. Both electric fields are assumed to be linearly polarized in the same direction. Delay times $\tau_{\mathrm{P}}$ and $\tau_{\mathrm{L}}$ for probe and pump pulses, respectively, are introduced to ensure the time-invariance in final equations. Further on through this paper the pump field arrival time will be taken as reference one, therefore: $\tau_{\mathrm{L}}=0$ in all calculations. Field frequencies are assumed to be near to the optical transition of the system, in the sense that absolute values of frequency detunings for pump and probe pulses from resonance are considerably less in comparison to corresponding resonance frequency of the twolevel system.

Non-perturbed Hamiltonian of the two-level system is defined as

$$
\widehat{H}_{0}=\frac{1}{2}\left[\begin{array}{ll}
0 & 0 \\
0 \hbar & \omega_{21}
\end{array}\right],
$$

whereas perturbation due to electric field dipole interaction with the laser field is written as follows:

$$
\widehat{H}_{1}=-E(t)\left[\begin{array}{cc}
0 & \mu \\
\mu^{*} & 0
\end{array}\right],
$$

where matrix element $\mu$ stands for the dipole transition in two-level system. Dynamics of the system under consideration now is given by evolution of Liouville equation of the density matrix, as follows:

$$
\frac{\partial}{\partial t} \hat{\rho}=\left[\hat{\rho}, \widehat{H}_{0}+\widehat{H}_{1}\right] .
$$

Here we may recall some points of the well-known pump-probe spectroscopy. Specifically, in such measurements pump beam induces population and polarization in the sample, whereas weak probe pulse is used to test these changes. Usually, fields are directed to the sample at different angles, and two polarization waves interfere to produce a grating with period $\mathbf{h}=\left|\mathbf{k}_{\mathrm{L}}-\mathbf{k}_{\mathrm{P}}\right|$ in $k$-space. Diffraction on this grating results in energy transfer from the pump beam to the probe one. In our simulations it is assumed that moduli of wave vectors $\left|\mathbf{k}_{\mathrm{L}}\right|,\left|\mathbf{k}_{\mathrm{P}}\right|$ are equal and modulation of the refraction index is quite small. Therefore, we restrict ourselves to the first order of diffraction only, by defining polarization amplitudes $Q_{\mathrm{L}}, Q_{\mathrm{P}}$, and $Q_{\mathrm{L} 2 \mathrm{P}}$ (propagating at directions $\mathbf{k}_{\mathrm{L}}, \mathbf{k}_{\mathrm{P}}$, and $2 \mathbf{k}_{\mathrm{L}}-\mathbf{k}_{\mathrm{P}}$, respectively), and expanding polarization $\rho_{12}$ and population $n=\rho_{22}-\rho_{11}$ in the following manner [15]:

$$
\begin{gathered}
\rho_{12}=Q_{\mathrm{L}} \mathrm{e}^{\mathrm{i} \mathbf{k}_{\mathrm{L}} \mathbf{r}}+Q_{\mathrm{P}} \mathrm{e}^{\mathrm{i} \mathbf{k}_{\mathrm{P}} \mathbf{r}}+Q_{\mathrm{L} 2 \mathrm{P}} \mathrm{e}^{\mathrm{i} 2 \mathbf{k}_{\mathrm{L}} \mathbf{r}-\mathbf{k}_{\mathrm{P}} \mathbf{r}}, \\
n=n_{0}+n_{1} \mathrm{e}^{\mathrm{i} \mathbf{H r}}+n_{-1} \mathrm{e}^{-\mathrm{i} \mathbf{H r}} .
\end{gathered}
$$

(Note that $n_{-1}=n_{1}^{*}$.) After substituting expressions (5) into Liouville equations one gets the following equations describing evolution of this two-level system:

$$
\begin{aligned}
& \frac{\partial}{\partial t} n_{0}= \mathrm{i} \Lambda_{\mathrm{L}}^{*} Q_{\mathrm{L}}-\mathrm{i} \Lambda_{\mathrm{L}} Q_{\mathrm{L}}^{*}+\mathrm{i} \Lambda_{\mathrm{P}}^{*} Q_{\mathrm{P}}-\mathrm{i} \Lambda_{\mathrm{P}} Q_{\mathrm{P}}^{*} \\
&-\frac{n_{0}-n_{0}(0)}{T_{1}} \\
& \frac{\partial}{\partial t} n_{1}= \mathrm{i} \Lambda_{\mathrm{L}}^{*} Q_{\mathrm{P}}-\mathrm{i} \Lambda_{\mathrm{P}} Q_{\mathrm{L}}^{*}+\mathrm{i} \Lambda_{\mathrm{L}} Q_{\mathrm{L} 2 \mathrm{P}}-\frac{n_{1}}{T_{1}} \\
& \frac{\partial}{\partial t} Q_{\mathrm{L}}=\mathrm{i} \omega_{12} Q_{\mathrm{L}}+\frac{\mathrm{i}}{2} \Lambda_{\mathrm{L}} n_{0}+\frac{\mathrm{i}}{2} \Lambda_{\mathrm{P}} n_{-1}-\frac{Q_{\mathrm{L}}}{T_{2}}, \\
& \frac{\partial}{\partial t} Q_{\mathrm{P}}=\mathrm{i} \omega_{12} Q_{\mathrm{P}}+\frac{\mathrm{i}}{2} \Lambda_{\mathrm{L}} n_{1}+\frac{\mathrm{i}}{2} \Lambda_{\mathrm{P}} n_{0}-\frac{Q_{\mathrm{P}}}{T_{2}},
\end{aligned}
$$




$$
\frac{\partial}{\partial t} Q_{\mathrm{L} 2 \mathrm{P}}=\mathrm{i} \omega_{12} Q_{\mathrm{L} 2 \mathrm{P}}+\frac{\mathrm{i}}{2} \Lambda_{\mathrm{L}} n_{1}-\frac{Q_{\mathrm{L} 2 \mathrm{P}}}{T_{2}},
$$

where terms with characteristic constants $T_{1}$ and $T_{2}$ are introduced to account for population and polarization relaxation, respectively. $n_{0}(0)$ is initial population of the system (in our simulations $n_{0}(0)=$ -1 , as the system is assumed to be in ground state), $\Lambda_{\mathrm{L}, \mathrm{P}}=\mu \varepsilon_{\mathrm{L}, \mathrm{P}}(t) / \hbar$ corresponds to the Rabi frequencies of pump and probe fields.

To exclude the dominant "fast" oscillations at frequencies $\omega_{\mathrm{L}}$ and $\omega_{\mathrm{P}}$ from polarization $Q_{\mathrm{L}}, Q_{\mathrm{L} 2 \mathrm{P}}$, and $Q_{\mathrm{P}}$, those are replaced with $Q_{\mathrm{L}}=Q_{\mathrm{L}}^{s} \mathrm{e}^{\mathrm{i}\left[-\omega_{\mathrm{L}}\left(t-\tau_{\mathrm{L}}\right)\right]}$, $Q_{\mathrm{L} 2 \mathrm{P}}=Q_{\mathrm{L} 2 \mathrm{P}}^{s} \mathrm{e}^{\mathrm{i}\left[-2 \omega_{\mathrm{L}}\left(t-\tau_{\mathrm{L}}\right)+\omega_{\mathrm{P}}\left(t-\tau_{\mathrm{P}}\right)\right]}$, and $Q_{\mathrm{P}}=$ $Q_{\mathrm{P}}^{s} \mathrm{e}^{\mathrm{i}\left[-\omega_{\mathrm{P}}\left(t-\tau_{\mathrm{P}}\right)\right]}$. Then, using rotating wave approximation, equations for material variables (polarization and population) can be written as

$$
\begin{gathered}
\frac{\partial}{\partial t} n_{0}=\mathrm{i} \Lambda_{\mathrm{L}} Q_{\mathrm{L}}^{s}-\mathrm{i} \Lambda_{\mathrm{L}}^{*} Q_{\mathrm{L}}^{s *}+\mathrm{i} \Lambda_{\mathrm{P}} Q_{\mathrm{P}}^{s}-\mathrm{i} \Lambda_{\mathrm{P}}^{*} Q_{\mathrm{P}}^{s *} \\
-\frac{n_{0}-n_{0}(0)}{T_{1}}
\end{gathered}
$$$$
\frac{\partial}{\partial t} n_{1}=\mathrm{i}\left(-\Delta_{\mathrm{P} 12}+\Delta_{\mathrm{L} 12}\right) n_{1}+\mathrm{i} \Lambda_{\mathrm{L}} Q_{\mathrm{P}}^{s}-\mathrm{i} \Lambda_{\mathrm{P}} Q_{\mathrm{L}}^{s *}
$$$$
-\mathrm{i} \Lambda_{\mathrm{L}} Q_{\mathrm{L} 2 \mathrm{P}}^{s}-\frac{n_{1}}{T_{1}}
$$

$$
\begin{aligned}
& \frac{\partial}{\partial t} Q_{\mathrm{L}}^{s}=-\mathrm{i} \Delta_{\mathrm{L} 12} Q_{\mathrm{L}}^{s}+\frac{\mathrm{i}}{2} \Lambda_{\mathrm{L}} n_{0}+\frac{\mathrm{i}}{2} \Lambda_{\mathrm{P}} n_{-1}-\frac{Q_{\mathrm{L}}^{s}}{T_{2}}, \\
& \frac{\partial}{\partial t} Q_{\mathrm{P}}^{s}=-\mathrm{i} \Delta_{\mathrm{P} 12} Q_{\mathrm{P}}^{s}+\frac{\mathrm{i}}{2} \Lambda_{\mathrm{L}} n_{1}+\frac{\mathrm{i}}{2} \Lambda_{\mathrm{P}} n_{0}-\frac{Q_{\mathrm{P}}^{s}}{T_{2}}
\end{aligned}
$$

$$
\begin{aligned}
\frac{\partial}{\partial t} Q_{\mathrm{L} 2 \mathrm{P}}^{s}= & -\mathrm{i}\left(2 \Delta_{\mathrm{L} 12}-\Delta_{\mathrm{P} 12}\right) Q_{\mathrm{L} 2 \mathrm{P}}^{s}+\frac{\mathrm{i}}{2} \Lambda_{\mathrm{L}} n_{1} \\
& -\frac{Q_{\mathrm{L} 2 \mathrm{P}}^{s}}{T_{2}}
\end{aligned}
$$

where $\Delta_{\mathrm{L} 12}=\omega_{\mathrm{L}}-\omega_{12}$ and $\Delta_{\mathrm{P} 12}=\omega_{\mathrm{P}}-\omega_{12}$ are detunings of the pump and probe pulses from resonance. In our simulations they are assumed to be equal in dispersed pump-probe measurement (see below), when spectrum of the probe pulse is considered as supercontinuum. In this case the detuning of the probe pulse is not crucial, provided probe spectrum is taken sufficiently broad.

Two kinds of pump-probe experiments are known these are dispersed and two-colour pump-probe experiments. In the latter case both pulses are of the same duration. Transmission differences can be calculated at various delay times between probe and pump beams, also when pump and probe are off resonance. Difference spectrum in this case reads:

$$
\Delta A(\tau) \sim \int\left|\Lambda_{\mathrm{P}}(\tau)\right|^{2} \mathrm{~d} t-\int\left|\Lambda_{\mathrm{P}}^{0}(\tau)\right|^{2} \mathrm{~d} t .
$$

Here $\tau$ denotes the delay time between pump and probe fields. $\Lambda_{\mathrm{P}}$ and $\Lambda_{\mathrm{P}}^{0}$ stand for the cases when pump pulse is turned on and off, respectively. (In fact, due to calculation problems, $\Lambda_{\mathrm{P}}^{0}$ is evaluated for the long delay of the pump pulse.)

The evolution of probe field is expressed using Maxwell equation:

$$
\begin{gathered}
\frac{\partial}{\partial z} \Lambda_{\mathrm{P}} \sim-\mathrm{i} Q_{\mathrm{P}}^{s}\left(\Lambda_{\mathrm{L}} \neq 0, \Lambda_{\mathrm{P}}, \tau\right), \\
\frac{\partial}{\partial z} \Lambda_{\mathrm{P}}^{0} \sim-\mathrm{i} Q_{\mathrm{P}}^{s}\left(\Lambda_{\mathrm{L}}=0, \Lambda_{\mathrm{P}}, \tau\right) .
\end{gathered}
$$

When dispersed pump-probe experiment is investigated, one calculates differential transmission spectrum of the probe field. It can be written as follows:

$\Delta A(\omega, \tau) \sim\left|\int \Lambda_{\mathrm{P}}(\tau) \mathrm{e}^{-\mathrm{i} \omega t} \mathrm{~d} t\right|^{2}-\left|\int \Lambda_{\mathrm{P}}^{0}(\tau) \mathrm{e}^{-\mathrm{i} \omega t} \mathrm{~d} t\right|^{2}$.

Runge-Kutta method of fourth order is used to simulate the Eqs. (11)-(15).

\subsection{Two-level system in the representation of \\ "dressed" states}

Before presenting results of the Stark effect manifestation in differential absorption spectra, it is instructive to have a look at physics of the two-level system in a strong near-resonant $\mathrm{cw}$ laser field. It is well known that Rabi oscillations, i.e. sinusoidal time evolution of the material variables (population and polarization) results in the splitting of the quantum states of composite light-matter system in the dressed-state representation (see e. g. $[10,17,18]$ and references therein).

Due to strong interaction between field and two-level quantum system, the superposition of field and quantum system is approached. In this case Schrödinger equation

$$
\mathrm{i} \hbar \frac{\partial}{\partial t} \psi(\mathbf{r}, t)=H \psi(\mathbf{r}, t)
$$

for stationary system Hamiltonian $H$ can be solved expressing the wave function $\psi(\mathbf{r}, t)$ as superposition:

$$
\psi(\mathbf{r}, t)=C_{1}(t)|g\rangle \mathrm{e}^{-\mathrm{i} \omega_{1} t}+C_{2}(t)|e\rangle \mathrm{e}^{-\mathrm{i} \omega_{2} t},
$$


where $C_{j}(t)$ represents probability amplitude to find system in state $|j\rangle, j=(g, e)$. Assuming that modulus of coefficient $C_{j}(t)$ is constant, $\left|C_{j}(t)\right|=$ const, and using appropriate boundary conditions (details of the full solution of this problem see in [18]) the wave vectors $|g\rangle_{ \pm}$and $|e\rangle_{ \pm}$describing superposition of the compound field and matter system are found. Probabilities to find the system in "dressed" states reads:

$$
\left|\langle\psi \mid g\rangle_{ \pm}\right|^{2}=\frac{|\Lambda|^{2}}{2 \Lambda^{\prime}\left(\Lambda^{\prime} \mp \Delta\right)},\left|\langle\psi \mid e\rangle_{ \pm}\right|^{2}=\frac{\Lambda^{\prime} \mp \Delta}{2 \Lambda^{\prime}} .
$$

Here $\Lambda^{\prime}=\sqrt{\Lambda^{2}+\Delta^{2}}$ is a generalized Rabi frequency.

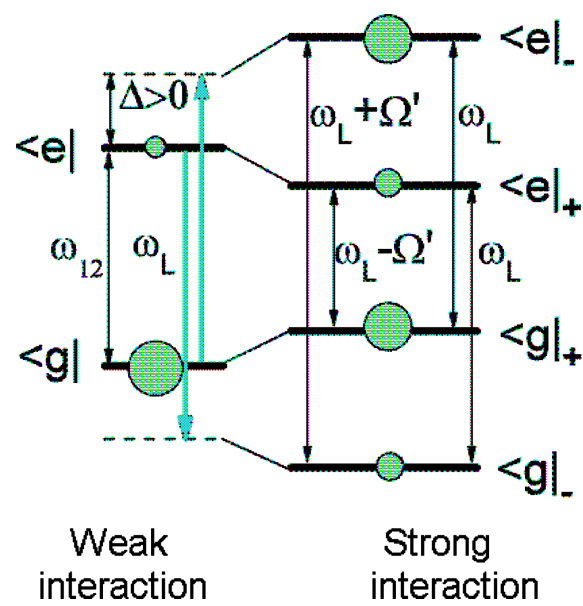

Fig. 1. Dressed atomic states in the cases of weak and strong interaction, corresponding to the case $\Delta>0$. Grey circles mark the population of quantum state.

Schematic representation of the "dressed" by the electromagnetic field two-level system is drawn in Fig. 1 for weak- and strong-field cases. Possible dipole transitions at three frequencies $\omega, \omega+\Lambda^{\prime}$, and $\omega-\Lambda^{\prime}$ are marked here by arrows.

Differential absorption spectra corresponding to the transition to "dressed" states can be easily calculated from Eqs. (11)-(15). In stationary case a linear set of equations for separate Fourier components reads as follows:

$$
\begin{aligned}
& \left(\omega_{\mathrm{P}}-\omega_{12}-\frac{\mathrm{i}}{T_{2}}\right) Q_{\mathrm{P}}=\Lambda_{\mathrm{P}} n(0)+\Lambda_{\mathrm{L}} n\left(\omega_{\mathrm{P}}-\omega_{\mathrm{L}}\right), \\
& \left(\omega_{\mathrm{P}}-\omega_{12}\right) n\left(\omega_{\mathrm{P}}-\omega_{\mathrm{L}}\right)=2\left(\Lambda_{\mathrm{L}}^{*} Q_{\mathrm{P}}-\Lambda_{\mathrm{P}} Q_{\mathrm{L}}^{*}-\Lambda_{\mathrm{L}} Q_{\mathrm{L} 2 \mathrm{P}}^{*}\right), \\
& \left(2 \omega_{\mathrm{L}}-\omega_{\mathrm{P}}-\omega_{12}+\frac{\mathrm{i}}{T_{2}}\right) Q_{\mathrm{L} 2 \mathrm{P}}^{*}=-2 \Lambda_{\mathrm{L}}^{*} n\left(\omega_{\mathrm{P}}-\omega_{\mathrm{L}}\right) .
\end{aligned}
$$

The solutions for polarization at directions $\mathbf{k}_{\mathrm{P}}$ and $2 \mathbf{k}_{\mathrm{L}}-\mathbf{k}_{\mathrm{P}}$ are:

$$
\begin{aligned}
& Q_{\mathrm{P}}= \\
& \begin{array}{l}
=\frac{\Lambda_{\mathrm{P}} n_{0}}{D}\left[\left(2 \omega_{\mathrm{L}}-\omega_{\mathrm{P}}-\omega_{12}+\frac{\mathrm{i}}{T_{2}}\right)\left(\omega_{\mathrm{P}}-\omega_{\mathrm{L}}-\frac{\mathrm{i}}{T_{1}}\right)\right. \\
\left.\quad+2 \Lambda_{\mathrm{L}}^{2}\left(1-\frac{2 \omega_{\mathrm{L}}-\omega_{\mathrm{P}}-\omega_{12}+\mathrm{i} / T_{2}}{\omega_{\mathrm{L}}-\omega_{12}+\mathrm{i} / T_{2}}\right)\right]
\end{array} \\
& Q_{\mathrm{L} 2 \mathrm{P}}^{*}=\frac{2 \Lambda_{\mathrm{L}}^{2} \Lambda_{\mathrm{P}} n_{0}}{D}\left(1-\frac{\omega_{\mathrm{P}}-\omega_{12}-\mathrm{i} / T_{2}}{\omega_{\mathrm{L}}-\omega_{12}+\mathrm{i} / T_{2}}\right)
\end{aligned}
$$

where $D$ is denominator expressed as

$$
\begin{aligned}
& D=\left(\omega_{\mathrm{P}}-\omega_{\mathrm{L}}\right) \\
& \times\left[-\left(\omega_{\mathrm{P}}-\omega_{\mathrm{L}}\right)^{2}+\left(\omega_{12}-\omega_{\mathrm{L}}\right)^{2}+4 \Lambda_{\mathrm{L}}^{2}+\frac{1}{T_{2}^{2}}+\frac{2}{T_{2} T_{1}}\right] \\
& +\frac{\mathrm{i}}{T_{2}}\left[2\left(\omega_{\mathrm{P}}-\omega_{\mathrm{L}}\right)^{2}+4 \Lambda_{\mathrm{L}}^{2}\right] \\
& +\frac{\mathrm{i}}{T_{1}}\left[\left(\omega_{\mathrm{P}}-\omega_{12}\right)\left(\omega_{\mathrm{P}}-2 \omega_{\mathrm{L}}+\omega_{12}\right)+\frac{1}{T_{2}^{2}}\right] .
\end{aligned}
$$

Differential absorption spectrum probed at $\mathbf{k}_{\mathrm{P}}$, calculated using these expressions, is drawn in the Fig. 2(a). This spectrum has well-known Mollow resonances [19] at frequencies which exactly match the transitions between "dressed" states:

$$
\omega_{\mathrm{P}}=\omega_{\mathrm{L}}, \omega_{\mathrm{P}}=\omega_{\mathrm{L}} \pm \Delta,
$$

where $\Delta=\sqrt{\left(\omega_{\mathrm{L}}-\omega_{12}\right)^{2}+4 \Lambda_{\mathrm{L}}^{2}+1 / T_{2}^{2}+2 /\left(T_{2} T_{1}\right)}$.
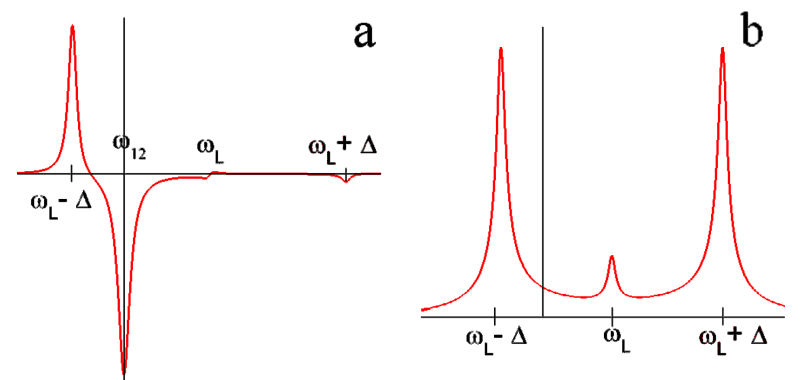

Fig. 2. Spectra in the limit of steady states: (a) differential absorption directed at $\mathbf{k}_{\mathrm{P}}$, (b) modulus of the polarization wave directed at $2 \mathbf{k}_{\mathrm{L}}-\mathbf{k}_{\mathrm{P}}$.

Additionally, negative dip at the resonance frequency $\omega_{12}$ is observed in Fig. 2(a) and it corresponds to reduced absorption from the ground to excited state due to the perturbation. 
On the other hand, it should be stressed that Stark shifts can be examined at the direction $2 \mathbf{k}_{\mathrm{L}}-\mathbf{k}_{\mathrm{P}}$, to which some part of the induced polarization is scattered due to the induced polarization grating. In Fig. 2(b) we plot the modulus of polarization scattered in this direction. In comparison to Fig. 2(a), absence of the negative dip at unperturbed resonance frequency $\omega_{12}$ should be stressed here.

\section{Results of numerical modelling and discussions}

Before we begin discussing results of numerical modelling, we will make some remarks on the parameters which will be used further throughout this paper. Firstly, as a population relaxation at the femtosecond to picosecond scale is negligible in the system we are interested in (QD), it has been excluded from simulations (i. e. $T_{1}$ is set to infinity). Phase relaxation time $T_{2}$ was varied in the region of $0.1-1 \mathrm{ps}$. In the case of dispersed pump-probe experiment probe pulse has to be wide enough to cover the whole line width, which is conditioned both by phase relaxation time and Rabi frequency. The latter parameter $\Lambda_{\mathrm{L}}$ was raised up to $60 \mathrm{ps}^{-1}$ (corresponding to the maximal used "area" $\Theta_{\mathrm{L}}=\int_{-\infty}^{\infty} \Lambda_{\mathrm{L}}(t) \mathrm{d} t$ of the pump pulse). Therefore probe pulse duration was set to 0.001 ps. As mentioned above, weak probe beam is used to probe induced absorption (transmission) changes, therefore its energy has to be weak and probe pulse area $\Theta_{\mathrm{P}}=\int \Lambda_{\mathrm{P}}(t) \mathrm{d} t$ has been set to 0.0001 . All spectra were calculated at zero delay - when pump and probe pulse coincided in time $\tau_{\mathrm{P}}=0$.

\subsection{Dispersed pump-probe experiment}

Usually the broad-band probe for the dispersed pumpprobe experiment is created by using supercontinuum generation in wide-band dielectrics: water, optical glass, and fibres. The part of the pump pulse is used for these purposes. Therefore, analysing this case it is reasonable to use the same carrier frequency both for the pump and probe pulses $\left(\omega_{\mathrm{L}}=\omega_{\mathrm{P}}\right)$. We start our analysis with the spectra, which are depicted for the fixed pump pulse area $\Theta_{\mathrm{L}}=70$, while changing detunings of the pump and probe pulses $\Delta_{\mathrm{L} 12}=\Delta_{\mathrm{P} 12}$ gradually from 20 to $60 \mathrm{ps}^{-1}$. Spectra calculated at three different phase relaxation times $\left(T_{2}=0.1,0.2\right.$, and $\left.0.5 \mathrm{ps}\right)$ are shown in Fig. 3(a-c). Due to the relatively short (as compared to the pump pulse) phase relaxation time, only negligible signatures of the OSE manifest here in the subfigures (a) and (b). Instead, these spectra are
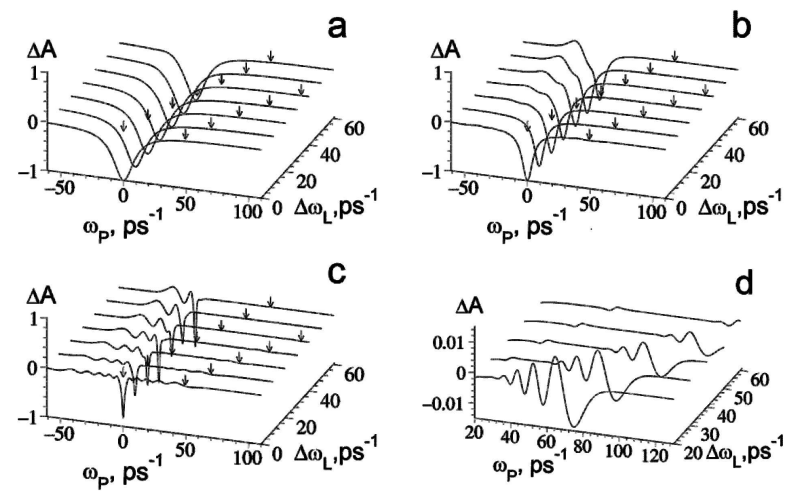

Fig. 3. Differential absorption spectra versus pump and probe pulse detuning from resonance depicted for different phase relaxation time: (a) $T_{2}=0.1 \mathrm{ps}$, (b) $0.2 \mathrm{ps,} \mathrm{(c)} 0.5 \mathrm{ps}$. Subfigure (d) shows magnified spectra for frequencies above the system resonance. The arrows mark frequencies $\omega_{\mathrm{L}}$ and $\omega_{\mathrm{L}}+\Lambda_{\mathrm{L}}, \tau_{\mathrm{L}}=0.5 \mathrm{ps}, \Theta_{\mathrm{L}}=70$.

dominated by the broad-band dip due to the reduced absorption at the two-level resonance. Nevertheless, increasing relaxation time $T_{2}$ leads to clear manifestation of the OSE in the spectra, which recall some features of the spectra in cw case shown in Fig. 2. In Fig. 3(b,c) these signatures become noticeable when Stark shift exceeds the width of the system spectra (i. e. when generalized Rabi frequency of the pump pulse becomes larger than the reciprocal phase relaxation time $\Lambda_{\mathrm{L}}>T_{2}^{-1}$ ) and manifest in an absorption increase in the low-energy side of the spectra. Note that the energy side on which more pronounced OSE is observable depends on the pump pulse frequency detuning from resonance. In our case pump pulse energy exceeds the difference between the energy levels in the two-level system. As a result OSE is clearly observed on the longer wavelength side with respect to system resonance, quite according to predictions made by using representation of the "dressed" states. The same features, though inversely signed and of considerably smaller amplitude, also occur on the shorter wavelength side. Figure 3(d) shows enlarged spectra corresponding to this wavelength region and displays both oscillations and an absorption decrease.

In addition to these observations, which are qualitatively similar to the ones shown in Fig. 2(a) and discussed in the subsection above, one aspect of the Stark shift spectroscopy should be mentioned here. Usually, line shifts of perturbed systems are determined by measuring positive peak of the "butterfly" feature in the differential absorption spectrum. It is noteworthy that in the case under consideration strong-field affected probe absorption undergoes changes both in position and shape (amplitude), as it is seen from Fig. 4. Specifically, a closer look at the (a) absorption and (b) 


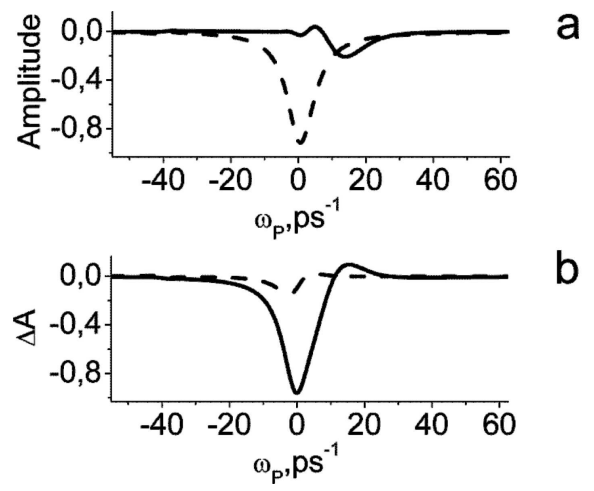

Fig. 4. Comparison of (a) absorption and (b) differential absorbtion spectra for weak $\left(\Theta_{\mathrm{L}}=10\right.$, dashed line $)$ and strong $\left(\Theta_{\mathrm{L}}=50\right.$, solid line) excitation cases. $T_{2}=0.5 \mathrm{ps}, \Delta \omega_{\mathrm{L}}=40 \mathrm{ps}^{-1}$.

differential absorption spectra shown in Fig. 4 reveals that "butterfly" in differential transmission spectrum is observed when Rabi frequency remains small. On the other hand, the "butterfly" splits into two peaks of opposite signs when the pump pulse amplitude increases. The first maximum matches the system resonance at frequency $\omega_{12}$ and the second one means the shifted line due to Stark effect. Therefore, when Stark shift exceeds the line width of the system, it can be measured more exactly from the positive peak position in the differential absorption spectrum unless direct measurement of the absorption spectrum is possible.
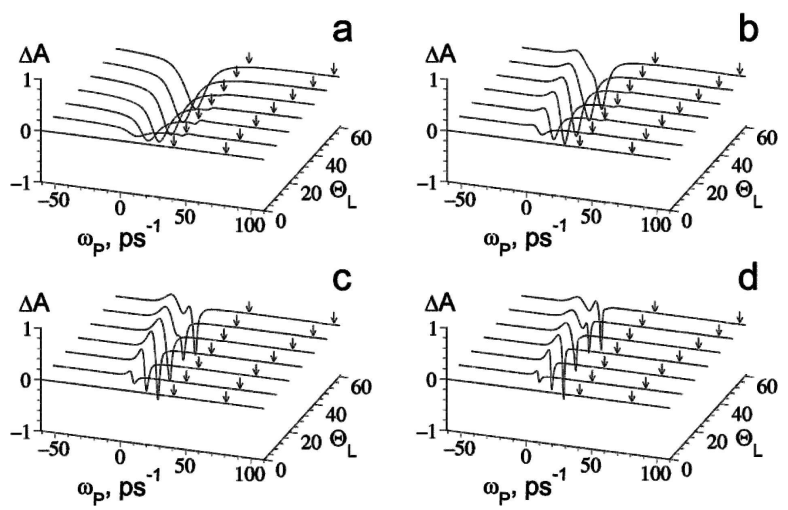

Fig. 5. Differential absorption spectra versus pump pulse "area" $\Theta_{\mathrm{L}}$, depicted for phase relaxation time (a) $T_{2}=0.1 \mathrm{ps}$, (b) $0.2 \mathrm{ps,}$ (c) $0.5 \mathrm{ps}$, (d) $1.0 \mathrm{ps}$. Pump field duration $\tau_{\mathrm{L}}=0.5 \mathrm{ps}$, its detuning from resonance $\Delta \omega_{\mathrm{L}}=40 \mathrm{ps}^{-1}$. The arrows here mark frequencies

$$
\omega_{\mathrm{L}} \text { and } \omega_{\mathrm{L}}+\Lambda_{\mathrm{L}} \text {. }
$$

Concluding this subsection we consider changes in the diferential absorption spectra when increasing pump pulse area, while keeping constant frequency detuning $\Delta_{\mathrm{L} 12}=\Delta_{\mathrm{P} 12}=40 \mathrm{ps}^{-1}$. The results at different phase relaxation time are shown in Fig. 5. Like in previous case, phase relaxation time $T_{2}$ has been chosen longer, equal, and shorter than pump pulse dura- tion. Pump pulse area has been varied in the region $\Theta=$ 1 .. 71. Other parameters have been left as in the case shown in Fig. 3. It is seen that differential absorption spectra for both cases depicted in Figs. 3-5 exhibit very similar changes both with respect to frequency detunig and phase relaxation time: here again small oscillations emerge on the shorter wavelength side for $\tau_{\mathrm{L}} \leq T_{2}$, and disappear when pump pulse duration is prolonged. The Stark shift in the differential absorption spectrum is seen only when generalized Rabi frequency exceeds the line width of homogenously broadened system, that is, $\Lambda_{\mathrm{L}}^{\prime}>T_{2}^{-1}$.

\subsection{Two-colour pump-probe spectroscopy}

Two-colour pump-probe experiment is easier to commit in practice than the dispersed one. Differential
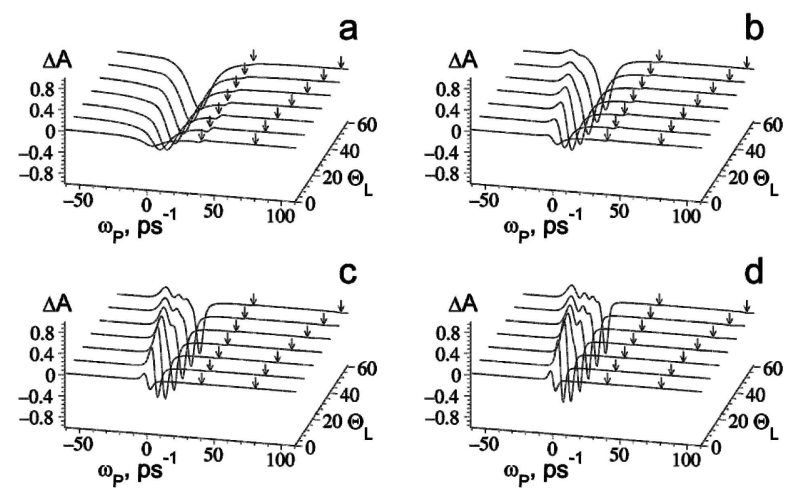

Fig. 6. Differential absorption spectra versus pump pulse "area" $\Theta_{\mathrm{L}}$, depicted at different values of phase relaxation time (a) $T_{2}=$ $0.1 \mathrm{ps}$, (b) $0.2 \mathrm{ps}$, (c) $0.5 \mathrm{ps}$, (d) 1 ps. Other parameters as in Fig. 5.

absorption spectra calculated for this case when both pump and probe pulses were taken as long as $0.5 \mathrm{ps}$ are shown in Fig. 6. In general terms, properties of the differential absorption spectra here remain the same as the ones discussed in the subsection above. Note the optimal value of the pump pulse area at which the most pronounced amplitude of the difference spectra appeared: $\Theta_{L} \simeq 30$. Finally, it is noteworthy to stress that the best choice for measuring Stark shifts is to set pump pulse duration close to phase relaxation time.

\subsection{Stark shifts in the spectra of diffracted radiation}

Described above peaks, slopes, and dips of the differential absorption spectra respond to the processes of different orders: linear (absorption and emission) and third-order (saturation and four-wave mixing). Therefore, they shade each other and make interpretation of the observed effects rather complicate. 

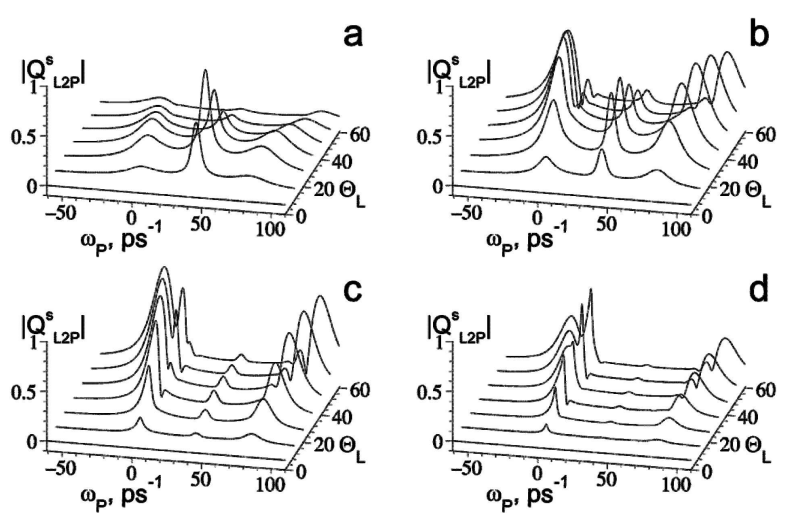

Fig. 7. Modulus spectra of polarization wave as obtained for dispersed pump-probe measurements at direction $2 \mathbf{k}_{\mathrm{L}}-\mathbf{k}_{\mathrm{P}}$ versus pump pulse "area" $\Theta_{\mathrm{L}}$, depicted for phase relaxation time (a) $T_{2}=$ $0.1 \mathrm{ps}$, (b) $0.2 \mathrm{ps}$, (c) $0.5 \mathrm{ps}$, (d) $1.0 \mathrm{ps}$. $\tau_{\mathrm{L}}=0.5 \mathrm{ps}, \Delta \omega_{\mathrm{L}}=40 \mathrm{ps}^{-1}$.
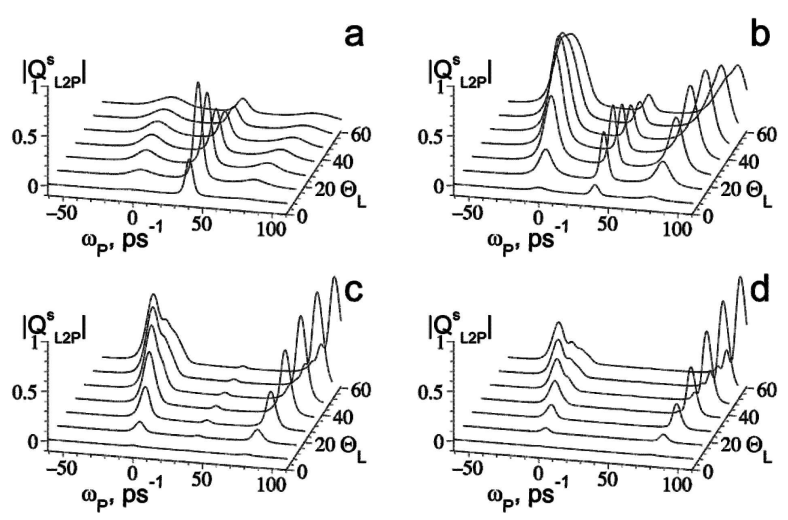

Fig. 8. Modulus spectra of polarization wave as obtained for twocolour pump-probe measurements at direction $2 \mathbf{k}_{\mathrm{L}}-\mathbf{k}_{\mathrm{P}}$ versus pump pulse "area" $\Theta_{\mathrm{L}}$, depicted for phase relaxation time (a) $T_{2}=$ $0.1 \mathrm{ps}$, (b) $0.2 \mathrm{ps}$, (c) $0.5 \mathrm{ps}$, (d) $1.0 \mathrm{ps}$. $\tau_{\mathrm{L}}=0.5 \mathrm{ps}, \Delta \omega_{\mathrm{L}}=40 \mathrm{ps}^{-1}$.

Thus it is useful to look the spectra at different (from pump and probe) direction, e. g. at $2 \mathbf{k}_{\mathrm{L}}-\mathbf{k}_{\mathrm{P}}$. Square of the modulus of the polarization wave given by Eq. (15) for dispersed and two-colour pump-probe measurement configurations are shown in Figs. 7 and 8. In both cases Mollow triplet lines [19] are clearly seen here. Fourwave mixing process is responsible for polarization in this direction. It appears as the pump and probe pulses interfere and is proportional to $\Lambda_{\mathrm{P}} \Lambda_{\mathrm{L}}^{2}$, as seen from steady state solution Eq. (27). Note that Rabi sidebands at frequencies $\omega_{12}$ and $\omega_{\mathrm{L}}+\Delta$ increase when increasing phase relaxation time (i. e. for stronger light-matter interaction case). Consequently, longer pulses, as compared to phase relaxation time, cause falling amplitudes of the Rabi sideband spectra, whereas distinct peak at the exciton resonance emerges in this case.

\section{Conclusions}

In this paper, we demonstrate the interference effects of the strong pump and weak probe in angleresolved differential pump-probe spectroscopy. The dressed atom approach in the frequency domain was used to interpret our findings. Numerics for both dispersed and two-colour configurations of pump-probe measurements, in general terms, reveal the characteristics of the well-known Mollow triplet spectrum, though it is kept hidden to great extent by different mixing contributions (four-wave mixing, bleaching due to the population changes, induced polarization waves), when measured in the direction of the probe beam. Nevertheless, decreasing line width of the two-level system beyond the generalized Rabi frequency of the pump pulse results in a more clear manifestation of OSE in the differential absorption spectrum and can be estimated from its positive peak position. On the other hand, clear Mollow triplet, with characteristic of this spectra dependences on excitation strength and relaxation time of coherent polarization, can be observed in "pure" fourwave mixing direction $2 \mathbf{k}_{\mathrm{L}}-\mathbf{k}_{\mathrm{P}}$ for measurements in both (dispersed and two-colour) configurations.

We should recall that Stark effect is of instantaneous response, and dressed states and quantum level splitting emerge only when system remains under excitation. This fact offers a possibility of all-optical femtosecond switch using two-photon absorption in threelevel system: e.g., recently a technique has been suggested where strong femtosecond laser beam switches off another laser beam of different wavelength due to the OSE in gases [21]. Our preliminary calculations show that extension of the model described above to threelevel system (specifically, by adding transition from exciton to bi-exciton state) offers the same possibility in semiconductor quantum dots, i.e. materials meeting the requirements of nanotechnology.

\section{References}

[1] E. Biolatti, R.C. Iotti, P. Zanardi, and F. Rossi, Quantum information processing with semiconductor macroatoms, Phys. Rev. Lett. 85(26), 5647-5650 (2000).

[2] P. Chen, C. Piermarocchi, and L.J. Sham, Control of exciton dynamics in nanodots for quantum operations, Phys. Rev. Lett. 87(6), 067401 (2001).

[3] A. Nazir, B.W. Lovett, and G.A.D. Briggs, Creating excitonic entanglement in quantum dots through the optical Stark effect, Phys. Rev. A 70(5), 052301 (2004). 
[4] A. Mysyrowicz, D. Hulin, A. Antonetti, A. Migus, W.T. Masselink, and H. Morkoc, "Dressed excitons" in a multiple-quantum-well structure: Evidence for an optical Stark effect with femtosecond response time, Phys. Rev. Lett. 56(25), 2748-2751 (1986).

[5] C. Weisbuch, M. Nishioka, A. Ishikawa, and Y. Arakawa, Observation of the coupled excitonphoton mode splitting in a semiconductor quantum microcavity, Phys. Rev. Lett. 69(23), 3314-3317 (1992).

[6] H. Kamada, H. Gotoh, J. Temmyo, T. Takagahara, and H. Ando, Exciton Rabi oscillation in a single quantum dot, Phys. Rev. Lett. 87(24), 246401 (2001).

[7] T.H. Stievater, Xiaoqin Li, D.G. Steel, D. Gammon, D.S. Katzer, D. Park, C. Piermarocchi, and L.J. Sham, Rabi oscillations of excitons in single quantum dots, Phys. Rev. Lett. 87(13), 133603 (2001).

[8] M. Chachišvilis, H. Fidder, and V. Sundström, Electronic coherence in pseudo two-collour pump-probe spectroscopy, Chem. Phys. Lett. 234(1-3), 141-150 (1995).

[9] P. Hamm, Coherent effects in femtosecond infrared spectroscopy, Chem. Phys. 200(3), 415-429 (1995).

[10] C. Cohen-Tannoudji, J. Dupont-Roc, and G. Grynberg, Atom-Photon Interactions: Basic Processes and Applications (Wiley, New York, 1992).

[11] E. Gaižauskas and L. Valkūnas, Coherent transients of pump-probe spectroscopy in two-level approximation, Opt. Commun. 109(1-2), 75-80 (1994).

[12] L. Seider, G. Stock, and W. Domcke, Nonpertubative approach to femtosecond pump-probe spectroscopy: General theory and application to multidimensional nonadiabatic photoisomerization processes, J. Chem. Phys. 103(10), 3998-4011 (1995).

[13] B. Wolfseder, L. Seider, G. Stock, and W. Domcke, Femtosecond pump-probe spectroscopy of electron transfer systems: A nonpertubative approach, Chem. Phys. 217(2-3), 275-287 (1997).

[14] E. Gaižauskas and L. Valkūnas, Femtosecond fourwave mixing spectroscopy of molecular aggregates, J. Phys. Chem. B, 101(37), 7321-7326 (1997).

[15] E. Gaižauskas, A. Beržanskis, and K.-H. Feller, Effects of non-Markovian relaxation in the femtosecond differential absorption spectroscopy, Chem. Phys. 235(1-3), 123-130 (1998).

[16] J.-S. Park and T. Joo, Coherent interactions in femtosecond transient grating, J. Chem. Phys. 120(11), 5269-5274 (2004).

[17] C. Cohen-Tannoudji and S. Reynaud, Dressed-atom description of resonance fluorescence and absorption spectra of a multilevel atom in an intense laser beam, J. Phys. B 10(3), 345-363 (1977).

[18] R. Boyd, Nonlinear Optics (Academic Press, San Diego, USA, 1992).

[19] B.R. Mollow, Stimulated emission and absorption near resonance for driven systems, Phys. Rev. A 5(5), 22172222 (1972).

[20] F.Y. Wu, S. Ezekiel, M. Ducloy, and B.R. Mollow, Observation of amplification in a strongly driven two-level atomic system at optical frequencies, Phys. Rev. Lett. 38(19), 1077-1080 (1977).

[21] D.D. Yavuz, All-optical femtosecond switch using twophoton absorption, Phys. Rev. A 74(5), 053804 (2006).

\title{
ŠTARKO EFEKTO APRAIŠKOS SKIRTUMINĖS SUGERTIES SPEKTRUOSE
}

\author{
A. Savickas, E. Gaižauskas \\ Vilniaus universitetas, Vilnius, Lietuva
}

\section{Santrauka}

Teoriškai tiriami skirtuminiai kvantinių taškų, pasižyminčių stipria sąveika su rezonansiniu elektromagnetiniu (EM) lauku, skirtuminès sugerties spektrai. Modeliuojama dviejų lygmenu kvantinè sistema, esanti nedideliu kampu susikertančių dvieju (kaupinančio ir zonduojančio) lazerinių pluoštų lauke. Kvantinèje sistemoje skirtingomis kryptimis indukuota poliarizacija įvertinta iš Liuvilio (Liouville) lygties tankio matricai ir skaičiuojama netai- kant ịprastų žadinimo ir zondavimo spektroskopijai trikdžių teorijos artinių. Apskaičiuoti skirtuminiai spektrai interpretuojami remiantis žinomu apvilktujų būsenų (angl. dressed states) modeliu, aprašančiu energijos lygmenų poslinkius stipriame EM lauke. Parodyta, kad EM lauko ir kvantinès sistemos sąveikos stiprị charakterizuojantį spektrą (Mollow tripletą) patogiausia stebèti ne zonduojančio pluošto kryptimi. 